\title{
Gender Difference in the Role of Posterodorsal Amygdala on the Regulation of Food Intake, Adiposity and Immunological Responses in Albino Wistar Rats
}

\author{
Venugopal Lalitha ${ }^{a}$ Gopal Krushna Pal ${ }^{a}$ Pravati Pal $^{a}$ Subash Chandra Parija ${ }^{b}$ \\ Sathish Babu Murugaiyan ${ }^{c}$ \\ Departments of a Physiology and ${ }^{\mathrm{b}}$ Microbiology, Jawaharlal Institute of Post-Graduate Medical Education and \\ Research (JIPMER), and ' Departments of Biochemistry, Mahatma Gandhi Medical College and Research Institute, \\ Puducherry, India
}

\section{Key Words}

Posterodorsal amygdala - Food intake · Humoral immunity . Body weight $\cdot$ Cell-mediated immunity

\begin{abstract}
Lesion of posterodorsal amygdala (PDA) has been known to produce hyperphagia and obesity in animal models. However, the influence of gender on food intake (FI), body weight (BW) and immunological parameters following PDA lesion is not yet known. The present work was carried out to study the effect of gender on the regulation of $\mathrm{Fl}, \mathrm{BW}$ and immunological parameters following lesions of PDA in albino Wistar rats. Twenty-four albino Wistar rats were divided equally into 2 groups - PDA group and control group - with 6 male and 6 female rats in each. In the experimental group, bilateral electrolytic lesion of the respective nuclei was performed by stereotaxy and post-lesion parameters were recorded. In the control group, sham lesion was made. Male-female difference in each parameter was determined. Following PDA lesion, Fl increased significantly in both male $(p<0.001)$ and female rats $(p<0.01)$ but the percentage increase in $\mathrm{Fl}$ was significantly more in female rats $(p<0.001)$. BW also increased in both the sexes but the increase in BW was signifi-
\end{abstract}

cant only in male rats $(p<0.05)$. Both male and female rats showed increase in the concentration of cluster of differentiation 4 (CD4), but the significant increase in CD4 concentration $(p<0.01)$ was seen only in male rats. CD8 concentration increased significantly in male rats $(p<0.05)$. The liver weight-BW ratio was significantly greater in female rats $(p<$ 0.001 ) following PDA lesions. Lesion of PDA results in accentuation of $\mathrm{Fl}$ and $\mathrm{BW}$ gain and activation of immunity. There is a gender difference in the inhibitory control of PDA on FI, BW and immunity.

(c) 2016 S. Karger AG, Basel

\section{Introduction}

Extra hypothalamic areas in the brain have been recently described to control food intake (FI) [1]. Mainly, the mesolimbic areas like amygdala, caudate nucleus, nucleus accumbens and septal areas are involved in the regulation of food and water intake [2]. Many of these areas influence FI partly through the hypothalamic centres and partly through the hormones or neurotransmitters secreted from limbic areas of brain that influence the hypothalamic feeding areas. Posterodorsal amygdala (PDA) is

\section{KARGER}

E-Mail karger@karger.com

www.karger.com/aon (c) 2016 S. Karger AG, Basel

0972-7531/16/0231-0006\$39.50/0
Dr. Gopal Krushna Pal, Professor and Head

Department of Physiology, JIPMER

Puducherry 605006 (India)

E-Maildrgkpal@gmail.com 
an important nuclear complex of amygdala, an essential component of limbic system that contains both medial and posterior group of amygdalar nuclei [3, 4]. PDA has been found to be involved in modulation of body weight (BW) and immunity in rats [5]. Lesion of amygdala produces hyperphagia and obesity in cats, dogs and monkeys, and recently, studies with rats has shown that the effective site is the PDA [6]. Even small bilateral lesions of the most posterodorsal aspects of the amygdala resulted in substantial weight gains [7]. Hence, amygdaloid nuclei are considered to be an important extra hypothalamic site in the central regulation of FI and BW [8-10].

Immunity is controlled mainly by humoral and neural factors [11]. Studies have shown that the medial amygdala and the hypothalamus (both medial and lateral) are involved in the regulation of cellular immune functions [5]. Though hypothalamic areas such as ventromedial hypothalamus (VMH), lateral hypothalamus and mesolimbic areas such as medial septum, amygdala and hippocampus influence immunity and metabolism via hypothalamo-pituitary-adrenal axis [12], the exact nature of immunomodulation has not been clearly assessed. Moreover, though gender difference in neural influences on control of BW has been observed, the effect of gender on central control of immunological responses has not been studied yet. Therefore, in the present study, we have planned to evaluate the gender difference in FI, adiposity and immunological responses following lesion of PDA in albino rats of Wistar strain.

\section{Methods}

Animals

After the approval of the research council and animal ethics committee of Jawaharlal Institute of Post-Graduate Medical Education and Research (JIPMER) (XVIII meeting of JIPMER Institutional Ethics Committee held on June 21, 2011; approval letter dated July 1,2011), a total of 24 (12 males and 12 females) institutebred healthy adult albino rats of Wistar strain weighing between 150 and $250 \mathrm{~g}$ were obtained for the study. The rats were housed in individual plastic cages with wire lids in the Animal Research Laboratory of Physiology department, JIPMER. A layer of husk was spread on the floor of the cages, and 12-hour light-dark cycle was maintained. Standard rodent chow and fresh tap water was available ad libitum. Rats were allowed to habituate in individual cages for 10 days before basal measurements were taken.

\section{Basal FI and BW Recordings}

After 10 days of habituation, $40 \mathrm{~g}$ of standard rodent chow and $100 \mathrm{ml}$ of fresh tap water ad libitum was provided every day. Daily FI and BW were measured for 1 week to determine the mean 24hour basal recordings.
Groups

Animals were divided randomly into the following 2 groups:

(1) PDA group (bilateral lesion made in the PDA; 6 males and 6 females).

(2) Control group (bilateral sham lesion of PDA; 6 males and $6 \mathrm{fe}-$ males).

\section{Procedures}

Anesthesia

Different anesthetic agents were used because the depth of anesthesia required for different procedures differed. For blood collection, we require only light anesthesia; hence ether was used as the anesthetic agent for blood collection. Ketamine $(0.25 \mathrm{ml} / 250 \mathrm{~g}$ BW) was injected intraperitoneally for making lesion and for sacrificing the animal; double the dose of ketamine was injected intraperitoneally as described by Dev et al. [13].

\section{Blood Collection}

For obtaining the basal immunological values, $1.5-2 \mathrm{ml}$ of blood was collected by Jugular venous puncture after 7 days of basal readings of FI and BW. For estimation of post-lesion immunological parameters, $5 \mathrm{ml}$ of blood was collected with the help of a syringe and needle by puncturing the left ventricle (cardiac puncture) during sacrifice of the animal before fixation of brain.

\section{Electrolytic Nuclear Lesion}

The stereotaxic procedure was performed as described by $\mathrm{Pal}$ et al. [14] for making the brain lesions. Bilateral electrolytic lesions of PDA were made by introducing electrodes into the nuclei on both sides according to the following co-ordinates (anterior: 0.33 $\mathrm{cm}$, lateral: $\pm 0.46 \mathrm{~cm}$, vertical: $0.72 \mathrm{~cm}$ ) obtained from the stereotaxic atlas of rat brain by Konig and Klippel [15] and allowing the anodal current of $0.5 \mathrm{~mA}$ to pass through the electrode. In animals undergoing sham lesions, all the above-mentioned steps were followed except that no current was passed.

\section{Parameters}

Physical Parameters

FI was measured in grams daily with an electronic weighing machine.

BW was measured in grams every alternate day with an electronic weighing machine for the entire period (4 weeks) of the study.

After blood collection and lesion/sham lesion procedure, the animals were allowed to recover from the stress of the intervention for a period of 14 days during which FI and BW were not measured.

\section{Immunological Parameters}

Immunological parameters namely cluster of differentiation 4 (CD4) concentration (pg/ml), CD8 concentration ( $\mathrm{ng} / \mathrm{ml})$, serum albumin (g/dl), serum globulin (g/dl), albumin-globulin (A-G) ratio, liver weight-BW (LW-BW) ratio, spleen weight-BW (SW-BW) ratio and serum immunoglobulin $M(\mathrm{Ig} M)(\mathrm{mg} / \mathrm{ml})$ were estimated following the standard procedures as practiced in the clinical laboratory of departments of Microbiology and Physiology of JIPMER, Pondicherry. Approximately, $5 \mathrm{ml}$ of blood was allowed to clot and then centrifuged to separate the serum. The serum samples were stored at $-20^{\circ} \mathrm{C}$ in labelled containers for subsequent analyses of the following parameters:

(1) CD4 concentration (Rat cluster of differentiation 4, CD4 ELISA kit, Genxbio, Cusabio) [16]. 
Table 1. FI, BW and immunological parameters in experimental male $(\mathrm{n}=6)$ and female $(\mathrm{n}=6)$ rats before (pre-lesion) and after (postlesion) PDA lesion

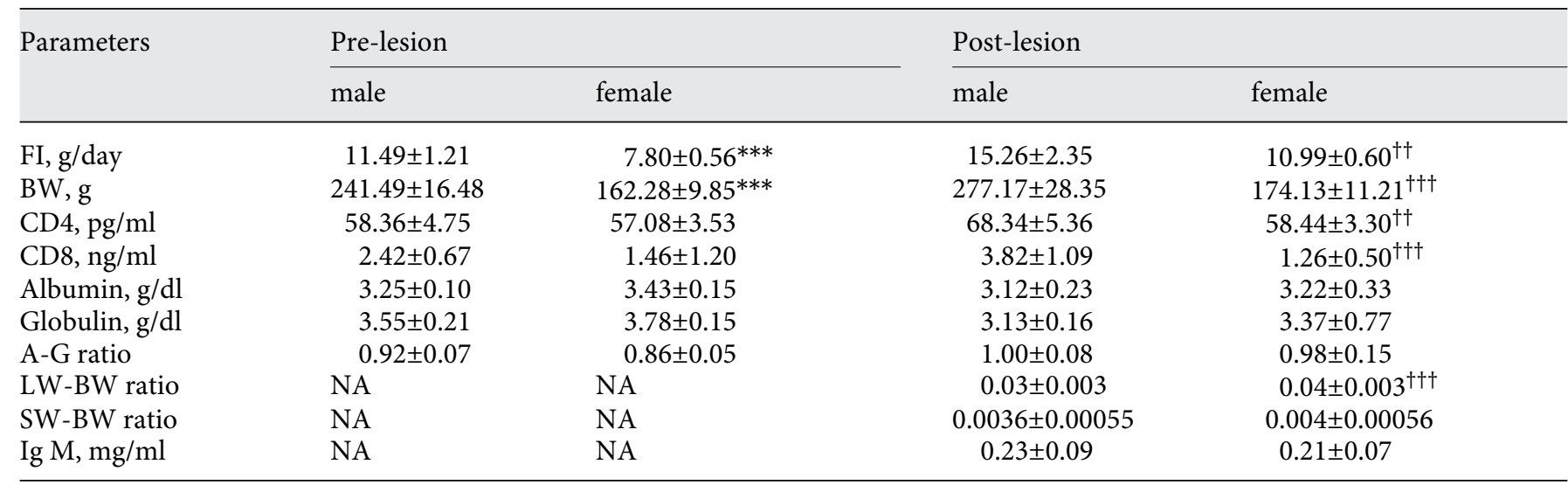

Data expressed are mean \pm SD.

* Represents comparison with pre-lesion experimental males, ${ }^{\dagger}$ represents comparison with post-lesion experimental males. The analysis of data was done by one-way ANOVA and post hoc by Tukey-Kramer test except for LW-BW ratio, SW-BW ratio and Ig M. Analysis of LW-BW ratio, SW-BW ratio and Ig M was done by Student's unpaired t test. ${ }^{*} \mathrm{p}<0.05 ;{ }^{* *} \mathrm{p}<0.01 ;{ }^{* * *} \mathrm{p}<0.001 ;{ }^{\dagger} \mathrm{p}<0.05$; ${ }^{\dagger \dagger} \mathrm{p}<0.01 ;{ }^{\dagger \dagger} \mathrm{p}<0.001$.

$\mathrm{NA}=$ Not applicable. Lesion means the lesion making needle electrode was introduced in to the PDA and current was passed.

(2) CD8 concentration (Rat cluster of differentiation 8, CD8 ELISA kit, Genxbio, Cusabio) [16].

(3) Serum albumin and globulin (Biuret method, Reagent kit adapted to Agappe Diagnostic, India) [17].

(4) Serum Ig M (Rat Ig M ELISA kit, Genxbio, Cusabio) [2]. Using serum albumin and globulin values, A-G ratio was calculated.

\section{Sacrifice of Animals}

After recording 4 weeks of post-interventional readings, all the animals were immunized on the 28th day with $1 \mathrm{ml}$ of sheep red blood cells [2]. Following this, all the animals were sacrificed on the 8 th day of immunization as per the standard procedure described by Pal et al. [14]. The liver and spleen were removed and weighed. LW-BW ratio and SW-BW ratio were measured.

\section{Statistical Analysis}

Data was expressed as mean \pm SD. Differences between means were compared by Student's t test. The differences among means were evaluated by one-way analysis of variance (ANOVA) using GraphPad InStat (version 3, USA) software. Post hoc test was performed by Tukey-Krammer multiple comparison test. The difference was considered statistically significant if $\mathrm{p}$ values were $<0.05$.

\section{Results}

\section{Basal Parameters}

Basal FI and BW of male rats $(\mathrm{n}=12)$ were $11.65 \pm$ $0.24 \mathrm{~g} /$ day and $251.19 \pm 3.73 \mathrm{~g}$ and in female rats $(\mathrm{n}=$
12), FI and BW were $7.53 \pm 0.38 \mathrm{~g} /$ day and $160.51 \pm$ $2.48 \mathrm{~g}$ (data not given in the table). While comparing the FI, there was a significant gender difference with male rats eating more than female rats $(\mathrm{p}<0.001)$. Similarly, while comparing the $\mathrm{BW}$, male rats weighed more than female rats $(\mathrm{p}<0.001$; table 1$)$. Control $(\mathrm{n}=12)$ and experimental rats $(\mathrm{n}=12)$ of PDA group had similar FI and $\mathrm{BW}$ and immunological parameters like $\mathrm{CD} 4$ concentration, CD8 concentration, albumin, globulin and A-G ratio did not show significant change before the start of experiment both in control and experimental rats of PDA group (table 2).

\section{Effect of Sham Lesion in Control Group}

After undergoing sham lesions, the control male and female rats did not show any significant change in FI, BW and immunological parameters namely CD4 concentration, $\mathrm{CD} 8$ concentration, albumin, globulin and A-G ratio from the pre-sham levels (table 3).

\section{Effect of PDA Lesion in Experimental Group}

Following PDA lesions, FI increased significantly in both males $(\mathrm{p}<0.001)$ and females $(\mathrm{p}<0.01)$ compared to their pre-lesion values (table 4 ), but the percentage increase in FI was significantly more $(\mathrm{p}<0.001)$ in female rats when compared to male rats (fig. 1). Both males and females showed an increase in BW, but the increase in 
Table 2. Comparison of FI, BW and immunological parameters of control (rats selected for sham lesion) and experimental (rats selected for PDA lesion) rats of PDA group before lesion

\begin{tabular}{lccc}
\hline Parameters & $\begin{array}{l}\text { Control rats } \\
(\mathrm{n}=12)\end{array}$ & $\begin{array}{l}\text { Experimental } \\
\text { rats }(\mathrm{n}=12)\end{array}$ & p value \\
\hline FI, g/day & $9.54 \pm 3.23$ & $9.64 \pm 2.60$ & 0.9342 \\
$\mathrm{BW}, \mathrm{g}$ & $209.83 \pm 72.24$ & $201.88 \pm 56.01$ & 0.7660 \\
$\mathrm{CD} 4, \mathrm{pg} / \mathrm{ml}$ & $58.37 \pm 1.66$ & $57.72 \pm 0.90$ & 0.2458 \\
$\mathrm{CD}, \mathrm{ng} / \mathrm{ml}$ & $1.98 \pm 1.38$ & $1.94 \pm 0.67$ & 0.9288 \\
Albumin, g/dl & $3.29 \pm 0.18$ & $3.34 \pm 0.13$ & 0.4437 \\
Globulin, g/dl & $3.40 \pm 0.50$ & $3.57 \pm 0.31$ & 0.4952 \\
A-G ratio & $1.03 \pm 0.12$ & $0.94 \pm 0.04$ & 0.1120 \\
\hline
\end{tabular}

Data expressed are mean \pm SD.

$\mathrm{p}<0.05$ was considered significant. Analysis of data was done by Student's unpaired t test. Control means the lesion making needle electrode was introduced in to the brain but current was not passed. Experimental means the lesion making needle electrode was introduced in to the PDA and current was passed.

Fig. 1. Male-female difference of mean percentage change in experimental and control rats of PDA group following lesion of PDA compared to their pre-lesion values. Lesion means the lesion making needle electrode was introduced in to the PDA and current was passed. Control means the lesion making needle electrode was introduced in to the brain but current was not passed. * Represents comparison with male experimental. ${ }^{*} \mathrm{p}<0.05 ;{ }^{* *} \mathrm{p}<0.01$; $^{* *} \mathrm{p}<$ 0.001 .

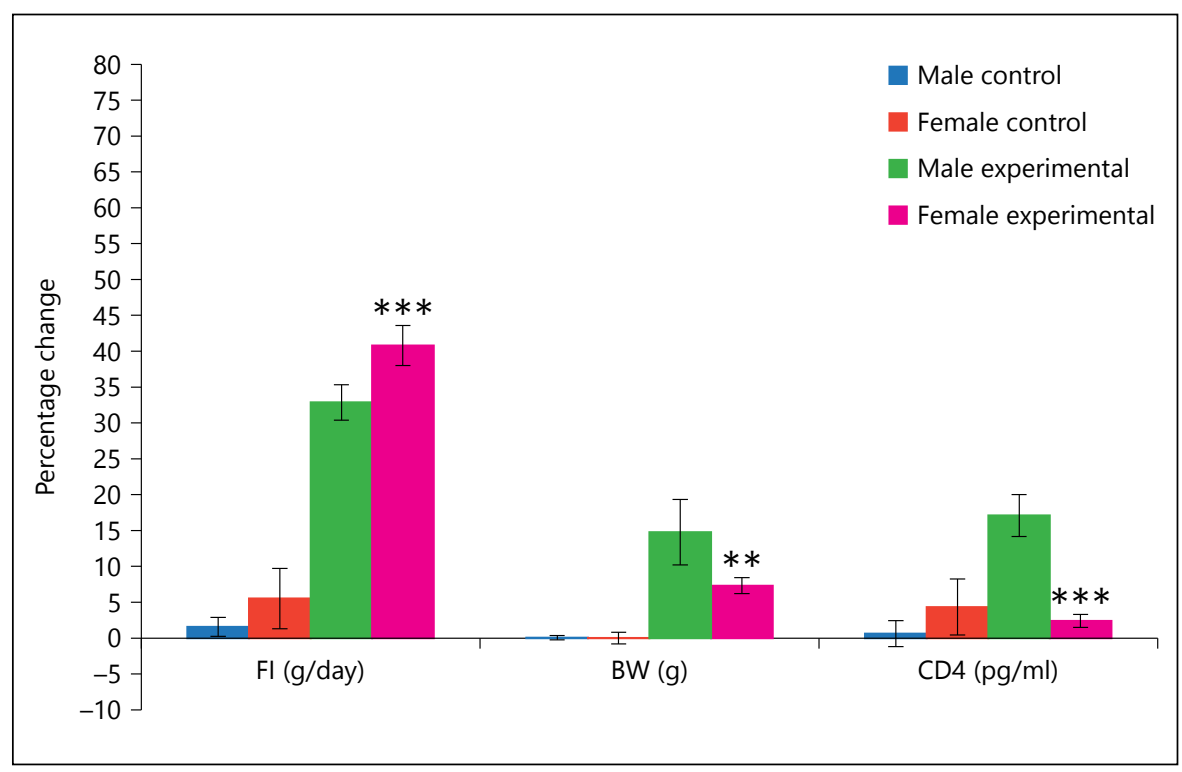

BW was significant $(\mathrm{p}<0.05)$ only in male rats (table 4$)$, and the percentage increase in BW was significantly more in male rats $(\mathrm{p}<0.01)$ when compared to female rats (fig. 1). Before the lesion, the FI and BW was significantly less in females $(\mathrm{p}<0.001)$ compared to male rats, and this gender difference in FI and BW was similar following PDA lesion (table 1).

After PDA lesion, both male and female rats showed increase in $\mathrm{CD} 4$ concentration, but the significant increase in CD4 concentration ( $<0.01)$ was seen only in male rats (table 4). Thus, a significant gender difference in the CD4 concentration was observed following PDA lesion ( $\mathrm{p}<0.01$ ), which was not present earlier (table 1 ). Also, the percentage increase in CD4 concentration was significantly higher in male rats $(\mathrm{p}<0.001)$ when compared to female rats (fig. 1). Following PDA lesion, there was no significant change in CD8 concentration in female rats, but CD8 concentration increased significantly in male rats $(\mathrm{p}<0.05)$. There was no significant gender difference observed in CD8 concentration before PDA lesion, but the CD8 concentration was significantly higher in male rats $(\mathrm{p}<0.001)$ when compared to female rats after PDA lesion (table 1). Though the levels of albumin and globulin decreased and A-G ratio in- 
Table 3. FI, BW and immunological parameters of control male $(n=6)$ and female $(n=6)$ rats before (pre-sham) and after $($ post-sham) PDA lesion

\begin{tabular}{|c|c|c|c|c|}
\hline \multirow[t]{2}{*}{ Parameters } & \multicolumn{2}{|l|}{ Control male } & \multicolumn{2}{|l|}{ Control female } \\
\hline & pre-sham & post-sham & pre-sham & post-sham \\
\hline FI, g/day & $11.83 \pm 0.96$ & $11.64 \pm 4.32$ & $7.26 \pm 0.74$ & $7.66 \pm 0.47$ \\
\hline BW, g & $260.91 \pm 8.58$ & $261.12 \pm 8.87$ & $158.75 \pm 16.66$ & $158.76 \pm 16.33$ \\
\hline $\mathrm{CD} 4, \mathrm{pg} / \mathrm{ml}$ & $59.54 \pm 5.09$ & $59.92 \pm 3.94$ & $57.19 \pm 3.10$ & $59.67 \pm 7.05$ \\
\hline $\mathrm{CD} 8, \mathrm{ng} / \mathrm{ml}$ & $2.96 \pm 0.99$ & $2.08 \pm 0.71$ & $1.01 \pm 0.28$ & $1.14 \pm 0.41$ \\
\hline Albumin, g/dl & $3.26 \pm 0.11$ & $3.14 \pm 0.24$ & $3.42 \pm 0.13$ & $3.33 \pm 0.22$ \\
\hline Globulin, g/dl & $3.04 \pm 0.78$ & $2.88 \pm 0.43$ & $3.75 \pm 0.72$ & $3.28 \pm 0.25$ \\
\hline A-G ratio & $1.11 \pm 0.37$ & $1.11 \pm 0.16$ & $0.94 \pm 0.19$ & $1.02 \pm 0.13$ \\
\hline LW-BW ratio & NA & $0.03 \pm 0.004$ & NA & $0.035 \pm 0.005$ \\
\hline SW-BW ratio & NA & $0.003 \pm 0.0005$ & NA & $0.0038 \pm 0.0008$ \\
\hline $\operatorname{Ig~M,~mg/ml~}$ & NA & $0.25 \pm 0.09$ & NA & $0.299 \pm 0.19$ \\
\hline
\end{tabular}

Data expressed are mean \pm SD.

The analysis of data was done by one-way ANOVA and post hoc by Tukey-Kramer test except for LW-BW ratio, SW-BW ratio and Ig M. Analysis of LW-BW ratio, SW-BW ratio and Ig $M$ was done by Student's unpaired t test.

$\mathrm{NA}=$ Not applicable. Sham means the lesion making needle electrode was introduced in to the brain but current was not passed.

Table 4. FI, BW and immunological parameters in experimental male $(n=6)$ and female $(n=6)$ rats before (pre-lesion) and after $($ postlesion) PDA lesion

\begin{tabular}{|c|c|c|c|c|}
\hline \multirow[t]{2}{*}{ Parameters } & \multicolumn{2}{|c|}{ Experimental male } & \multicolumn{2}{|c|}{ Experimental female } \\
\hline & pre-lesion & post-lesion & pre-lesion & post-lesion \\
\hline FI, g/day & $11.49 \pm 1.21$ & $15.26 \pm 2.35^{* * *}$ & $7.80 \pm 0.56$ & $10.99 \pm 0.60^{\dagger \dagger}$ \\
\hline $\mathrm{BW}, \mathrm{g}$ & $241.49 \pm 16.48$ & $277.17 \pm 28.35^{*}$ & $162.28 \pm 9.85$ & $174.13 \pm 11.21$ \\
\hline $\mathrm{CD} 4, \mathrm{pg} / \mathrm{ml}$ & $58.36 \pm 4.75$ & $68.34 \pm 5.36^{* *}$ & $57.08 \pm 3.53$ & $58.44 \pm 3.30$ \\
\hline $\mathrm{CD} 8, \mathrm{ng} / \mathrm{ml}$ & $2.42 \pm 0.67$ & $3.82 \pm 1.09^{*}$ & $1.46 \pm 1.20$ & $1.26 \pm 0.50$ \\
\hline Albumin, g/dl & $3.25 \pm 0.10$ & $3.12 \pm 0.23$ & $3.43 \pm 0.15$ & $3.22 \pm 0.33$ \\
\hline Globulin, g/dl & $3.55 \pm 0.21$ & $3.13 \pm 0.16$ & $3.78 \pm 0.15$ & $3.37 \pm 0.77$ \\
\hline$A-G$ ratio & $0.92 \pm 0.07$ & $1.00 \pm 0.08$ & $0.86 \pm 0.05$ & $0.98 \pm 0.15$ \\
\hline LW-BW ratio & NA & $0.03 \pm 0.003$ & NA & $0.04 \pm 0.003$ \\
\hline SW-BW ratio & NA & $0.0036 \pm 0.00055$ & NA & $0.004 \pm 0.00056$ \\
\hline $\mathrm{Ig} \mathrm{M}, \mathrm{mg} / \mathrm{ml}$ & NA & $0.23 \pm 0.09$ & NA & $0.21 \pm 0.07$ \\
\hline
\end{tabular}

Data expressed are mean \pm SD.

* Represents comparison with pre-lesion experimental males; ${ }^{\dagger}$ represents comparison with pre-lesion experimental females. The analysis of data was done by one-way ANOVA and post hoc by Tukey-Kramer test except for LW-BW ratio, SW-BW ratio and Ig M. Analysis of LW-BW ratio, SW-BW ratio and Ig M was done by Student's unpaired test. ${ }^{*} \mathrm{p}<0.05 ;{ }^{* *} \mathrm{p}<0.01 ;{ }^{* * *} \mathrm{p}<0.001 ;{ }^{\dagger} \mathrm{p}<$ $0.05 ;{ }^{\dagger \dagger} \mathrm{p}<0.01 ;{ }^{\dagger \dagger} \mathrm{p}<0.001$.

$\mathrm{NA}=$ Not applicable. Lesion means the lesion making needle electrode was introduced in to the PDA and current was passed.

creased in both sexes following PDA lesion, the changes were not significant. The LW-BW ratio was significantly greater in female rats $(\mathrm{p}<0.001)$ when compared to male rats, but no significant gender difference was observed in SW-BW ratio or Ig M following PDA lesion (table 1).

\section{Discussion}

Lesion of PDA has been known to produce hyperphagia and obesity in animal models. In our study also, bilateral PDA lesion resulted in a significant increase in FI and $\mathrm{BW}$ in all the animals. However, there was a gender 
difference with female rats eating significantly more food $(40.8 \%)$ compared to male rats (32.9\%). In spite of a significantly higher percentage increase in FI, percentage increase in BW gain was less in female rats $(7.3 \%)$ compared to male rats $(14.8 \%)$, and this difference was statistically significant $(\mathrm{p}<0.05)$. This indicated that the inhibitory nature of PDA on FI is more pronounced in females and its inhibition on BW gain is more marked in males. Thus the neural influence of PDA is different on each gender. The influence of PDA on FI and BW is the same as that of $\mathrm{VMH}$ indicating that PDA also has a stronger regulation of adiposity in male rats similar to that of $\mathrm{VMH}[18]$.

Important immunological parameters recorded in the present study are CD4 concentration, CD8 concentration, albumin, globulin, A-G ratio, LW-BW ratio, SW$\mathrm{BW}$ ratio and Ig M. CD4 is the expression and marker of T4 cells that are designated to be the inducer or helper cells in immunological development $[11,19]$. Following lesion of PDA, the CD4 concentration increased significantly in male rats depicting the immunosuppressive effect of PDA in male rats. This was corroborated with a significant increase in CD8 concentration in male rats $(\mathrm{p}<0.05)$, further establishing the cellular immunosuppressive effect of PDA. This shows that normally PDA causes suppression of cell-mediated immunity whereas lesion of PDA removes the inhibition on cell-mediated immunity and this activation of cellular immunological response is more in males following PDA lesion. Though not significant, the A-G ratio indicative of humoral immunity increased in the experimental rats following PDA lesion. This shows the normal humoral immunosuppressive effect of PDA in rats. This was corroborated with a significant increase in LW-BW ratio in female rats compared to male rats $(\mathrm{p}<0.001)$, further establishing the humoral immunosuppressive effect of PDA, which was more prominent in female rats. This shows that normally PDA suppresses humoral immunity whereas lesion of
PDA causes activation of humoral immunity, and this alteration in humoral immunological response was more in females following PDA lesion. These findings clearly depict the differential amygdalar neural control mechanisms on cell-mediated and humoral immunity in either gender. Hence, it appears that PDA controls the hypothalamic pituitary immunomodulation system via its inhibitory amygdalo-hypothalamic projections.

Our present work was aimed at examining the possible involvement of PDA in the control of FI, BW gain and immunity and the influence of gender on these parameters. We observed that normally PDA suppresses both cell-mediated and humoral immunity; therefore, PDA lesion resulted in activation of both cell-mediated and humoral immunity. The alteration in cellular immunological parameters was more in male rats, whereas the humoral immunological response was more in female rats. Hence, PDA, the key component of limbic system, is thought to be an extra hypothalamic structure involved in the control of adiposity, immunity and behavioral aspects of feeding via its influence on other mesolimbic structures. One of the probable mechanisms might be that PDA refines the VMH output on adiposity and immunity. However, further studies should be done to assess the nature of interaction between limbic and hypothalamic structures to ascertain the amygdalar control of hypothalamic regulation of adiposity and immunity.

\section{Conclusion}

The lesion of PDA results in accentuation of FI and BW gain and activation of immunity. Also, PDA shows a gender difference in its inhibitory control on FI, BW and immunity. Hence, PDA is an important extra-hypothalamic area for the regulation of adiposity, satiety and immunity in rat models.

\section{References}

1 Peters A, Pellerin L, Dallman MF, et al: Causes of obesity: looking beyond the hypothalamus. Prog Neurobiol 2007;81:61-88.

2 Nanda N, Pal GK, Koner BC: Effect of dopamine injection into caudate nucleus on immune responsiveness in rats: a pilot study. Immunol Lett 2005;96:151-153.

3 Pal GK: Limbic system; in Textbook of Medical Physiology, ed 2. New Delhi, Ahuja Publication, 2010, pp 909-916.
4 Frank JAE, Schwartzkroin PA, Phillips JO, et al: The limbic system; in Excitable Cells and Neurophysiology, ed 21. Philadelphia, WB Saunders, 1989, pp 693-717.

5 Jurkowski M, Trojniar W, Borman A, et al: Peripheral blood natural killer cell cytotoxicity after damage to the limbic system in the rat. Brain Behav Immun 2001;15:93-113.

6 King BM: Amygdaloid lesion-induced obesity: relation to sexual behavior, olfaction, and the ventromedial hypothalamus. Am J Physiol Regul Integr Comp Physiol 2006;291:1201-1214.

7 Rollins BL, King BM: Amygdala-lesion obesity: what is the role of the various amygdaloid nuclei? Am J Physiol Regul Integr Comp Physiol 2000;279:R1348-R1356.

8 Löscher W, Brandt C, Ebert U: Excessive weight gain in rats over extended kindling of the basolateral amygdala. Neuroreport 2003; 14:1829-1832. 
9 King BM, Arceneaux ER, Cook JT, et al: Temporal lobe lesion-induced obesity in rats: an anatomical investigation of the posterior amygdala and hippocampal formation. Physiol Behav 1996;59:843-848.

10 King BM, Cook JT, Rossiter KN, et al: Posterodorsal amygdaloid lesions in rats: longterm effects on body weight. Physiol Behav 1996;60:1569-1571.

11 Parija SC: Immune response; in Text Book of Microbiology \& Immunology, ed 1. Haryana, Elsevier, 2009, pp 139-148.

12 Gerhard M: Central nervous regulation of the hypothalamic-pituitary-adrenal axis and its impact on fertility, immunity, metabolism and animal welfare: a review. Arch Tienz Dummerstorf 2002;45:575-595.
13 Dev S, Pal P, Pal GK, et al: Role of ventromedial hypothalamus on energy homeostasis in albino rats: effect of gender. Indian J Physiol Pharmacol 2012;56:107-116.

14 Pal GK, Pravati P, Nivedita N, et al: Study of interaction of estrogen and dopamine injected into ventromedial hypothalamus on control of obesity in ovariectomized albino rats. Ann Neurosci 2007;14:8-12.

15 Konig JF, Klippel RA: The Rat Brain: A Stereotaxic Atlas of the Forebrain and Lower Parts of the Brain Stem. Baltimore, William \& Wilkins, 1963.

16 Jacobson JD, Ansari MA, Mansfield ME, et al: Gonadotropin-releasing hormone increases CD4 T-lymphocyte numbers in an animal model of immunodeficiency. J Allergy Clin Immunol 1999;104(3 pt 1):653-658.
17 Babu MS, Bobby Z, Habeebullah S: Increased inflammatory response and imbalance in blood and urinary oxidant-antioxidant status in South Indian women with gestational hypertension and preeclampsia. Clin Biochem 2012;45:835-838.

18 Lalitha V, Pal GK, Parija SC, et al: Effect of gender on food intake, adiposity and immunological responses following lesion of ventromedial hypothalamus in albino Wistar rats. Int J Clin Exp Physiol 2014;1:44-50.

19 Pal GK: Immunity; in Textbook of Medical Physiology, ed 2. New Delhi, Ahuja Publication, 2010, pp 109-128. 